\title{
Possibilités d'action publique pour une meilleure prise en compte des marchés domestiques du bois dans le cadre des accords de partenariat volontaire (APV)
}

\section{Synthèse des enseignements dégagés au Cameroun, en République démocratique du Congo, en Équateur, au Gabon et en Indonésie}

Paolo Omar Cerutti, Yustina Artati, Ahmad Dermawan, Alice Kelly, Guillaume Lescuyer, Elena Mejía, Krystof Obidzinski, Pablo Pacheco, Louis Putzel, Raphael Tsanga et Andrew Wardell

\section{Points essentiels}

- Le plan d'action FLEGT (Forest Law Enforcement, Governance and Trade, c'est-à-dire l'Accord sur l'application des réglementations forestières, la gouvernance et les échanges commerciaux) a été lancé en 2003 par l'Union européenne (UE) afin de lutter au niveau mondial contre l'exploitation forestière illégale. En particulier, le FLEGT vise à réduire le commerce du bois récolté illégalement entre l'UE et les pays partenaires producteurs de bois.

- Le FLEGT fonctionne grâce à deux principaux instruments : des accords commerciaux bilatéraux — appelés accords de partenariat volontaire (APV) — qui sont signés avec les pays producteurs qui le souhaitent, et le règlement sur le bois de l'Union européenne entré en vigueur en mars 2013. Ce règlement sur le bois de l'UE impose aux importateurs européens de faire preuve d'une diligence raisonnable lorsqu'ils s'approvisionnent en bois à l'étranger pour se prémunir contre les produits illégaux.

- À ce jour, six pays ont signé un APV. Cinq d'entre eux se sont même engagés à appliquer les dispositions de cet APV concernant la vérification de la légalité non seulement au bois exporté vers l'Europe, mais aussi à celui qui est commercialisé sur leurs marchés intérieurs. Ceci signifie que le bois récolté et vendu sur le marché domestique sera réglementé par des projets de permis nationaux dans le cadre de l'APV (c'est le système de garantie de la légalité du bois ou Timber Legality Assurance System, TLAS).

- Le Cameroun, la République démocratique du Congo (RDC), l'Équateur et l'Indonésie se caractérisent par un secteur domestique du bois important, dynamique et en grande partie informel, qui contribue à faire vivre localement des centaines de milliers d'utilisateurs de la forêt, notamment de petits paysans, des populations autochtones, des scieurs artisanaux, des négociants et des prestataires de service tels que les transporteurs. Le poids du secteur domestique au Gabon est moins important en raison de la faible population du pays et de la concentration des activités dans la capitale, Libreville. Dans la plupart des pays, l'emploi dans le secteur informel est comparable à celui du secteur du bois industriel formel, voire plus florissant. Le nombre estimé d'emplois informels fournis par le secteur varie beaucoup en fonction des pays, allant de 45000 au Cameroun à environ 1,5 million en Indonésie.

- Dans tous les pays étudiés, le secteur domestique du bois se caractérise par les activités de petits exploitants agricoles, de scieurs et de négociants qui possèdent rarement un permis de coupe légal, abattent et scient de petites quantités de bois à l'aide d'une tronçonneuse ou d'une scie mobile. Ce bois, qui n'est pas d'une grande qualité, est vendu sur les marchés domestiques ou de l'autre côté des frontières dans les pays voisins. II fait rarement l'objet d'une taxation formelle. D'après nos recherches cependant, au fur et à mesure que le produit avance dans la chaîne de production, 5 \% à 15 \% du total des coûts supportés par les opérateurs informels sont des pots-de-vin versés aux représentants des ministères, à la police locale, aux militaires et aux douaniers.

- En acceptant d'intégrer le secteur domestique dans leur système de garantie de la légalité du bois, les pays producteurs s'engagent à réformer en profondeur la gouvernance de tout le secteur forestier. Implicitement, ces réformes entraîneront des efforts de formalisation du secteur domestique par le suivi, la supervision et la vérification.

- Dans les pays du bassin du Congo et en Indonésie, ces réformes exigeront de redéfinir les cadres juridiques actuels qui sont surtout conçus autour des opérations industrielles de grande ampleur visant l'exportation et réalisées par des sociétés propriétaires de concessions forestières et politiquement puissantes. Dans tous les pays, la structure des lois existantes ne favorise pas les activités du marché domestique du bois à petite échelle. D'après les conclusions de Pro-Formal, il est nécessaire de faciliter et de simplifier l'accès à la ressource, de mettre au point et d'adopter des régimes fiscaux spécifiques au secteur domestique du bois (tels que des redevances, des taxes sur la transformation, le transport et la commercialisation), de faire 
progresser l'accès au crédit à des conditions favorables pour les petits opérateurs, d'améliorer la diffusion des informations auprès de ceux-ci et de créer des incitations en vue du respect de la loi.

- Dans les pays qui ont déjà signé un APV (Cameroun et Indonésie) ou qui continuent de négocier leur accord (RDC et Gabon), les usagers de la forêt et les négociants au niveau local ont souvent été absents des négociations nationales sur l'APV. Cela est dû à leur grand nombre, à leur éparpillement sur un vaste territoire et à une capacité d'organisation médiocre, ainsi qu'aux tensions qui existent souvent entre la volonté affichée des pouvoirs publics de les faire sortir du secteur informel et le comportement de fonctionnaires décentralisés à la recherche de rentes. Par conséquent, avant d'amorcer des réformes, des canaux de communication spécifiques doivent être mis en place pour les scieurs artisanaux et les négociants pour discuter de leurs besoins, des modes de gouvernance coutumière et des difficultés et attentes éventuelles par rapport à la formalisation.

- De même, les résultats montrent que le principe d'inclusion du gouvernement qui caractérisait les premiers débats sur l'APV a tendance à faiblir au fil du temps, car l'élan politique s'essouffle. Les instances gouvernementales autres que les ministères des forêts et de l'environnement peuvent être exclues des phases finales de la mise en œuvre, lorsque les réformes du cadre juridique doivent être adoptées. L'application de la loi pourrait alors s'avérer difficile, car ces instances sont susceptibles d'être réticentes à faire respecter des règles qu'elles n'ont pas contribué à mettre en place. L'implication d'autres ministères, en particulier ceux des finances, est nécessaire pour que soit constructif le débat national sur les répercussions financières du fonctionnement du système de garantie de la légalité du bois pour les opérateurs, surtout ceux de petite taille. Ces débats n'ont pas encore eu lieu. Cela pourrait entraîner l'échec de la mise en œuvre si l'on ne pèse pas dès le départ les avantages et les inconvénients du système (p. ex. les coûts pour les opérateurs).

- Les processus de formalisation engendrent des bénéfices comme des risques qui nécessitent en permanence une évaluation, un suivi et des mesures d'atténuation. Des études de cas sur la formalisation réalisées dans d'autres secteurs des ressources naturelles indiquent que même des processus bien intentionnés peuvent mener à l'exclusion, à la criminalisation, à des barrières à l'entrée et à la mainmise des élites sur la ressource. Cela pourrait nuire à l'environnement et porter un préjudice énorme aux petits exploitants et aux petits opérateurs. Bien conçue et bien suivie, la formalisation est susceptible d'accroître la transparence et de clarifier les droits des usagers, de réduire les conflits et de faire progresser les conditions de travail et les avantages économiques au niveau local.

\section{Introduction}

En 2003, I'UE a lancé le processus FLEGT pour enrayer l'exploitation forestière illégale et limiter les quantités de bois illégal qui entrent sur son territoire. Le FLEGT vise à favoriser des pratiques forestières durables, à améliorer la gouvernance forestière dans les pays producteurs de bois et à lutter contre la pauvreté. Les principaux instruments pour atteindre ces objectifs sont les APV et le règlement sur le bois de I'Union européenne. En substance, les APV sont des accords commerciaux en vertu desquels I'UE apporte une assistance technique et des formations pour aider à la mise en place de systèmes de garantie de la légalité du bois et veiller à l'efficacité des moyens de vérification de la légalité dans les pays producteurs partenaires. Le règlement sur le bois de l'Union européenne oblige tous les importateurs à faire preuve d'une diligence raisonnable concernant la légalité du bois qui arrive sur le marché de l'UE.

À ce jour, I'UE a ratifié six APV avec le Cameroun, la République centrafricaine, la République du Congo, le Ghana, I'Indonésie et le Libéria. D'autre part, un certain nombre de pays en Afrique, en Asie et en Amérique latine ont entamé des négociations (voir http://www.euflegt.efi.int/vpa-countries). Bien que la mise en œuvre des APV prenne plus de temps que prévu à l'origine, on reste confiant dans le fait que ces accords, conjointement avec le règlement sur le bois de l'Union européenne, contribueront à juguler l'exploitation forestière illégale dans les pays partenaires et à réduire la mise sur le marché européen de bois dont la commercialisation est illégale. des capacités garantiront non seulement efficacement l'intégrité du commerce direct du bois, mais consolideront aussi la gestion durable des forêts, et amélioreront la gouvernance forestière et les conditions de vie dans les pays producteurs. Ces mesures devraient permettre de réduire les inégalités par rapport au marché entre le secteur domestique des activités à petite échelle et le secteur industriel de grande ampleur orienté vers l'export.

Actuellement, les politiques nationales n'apportent pas une réponse adéquate aux problèmes associés à la croissance du secteur domestique, à son impact sur les forêts, à la législation correspondante et aux liens directs ou indirects avec l'exportation. Néanmoins, de nombreux pays dotés d'un APV (p. ex. le Cameroun, la République du Congo, le Ghana, I'Indonésie et le Libéria) ont élargi le champ de leur système de garantie de la légalité du bois pour inclure la totalité du bois produit et commercialisé sur le territoire national. Par conséquent, le processus FLEGT est destiné à attirer l'attention sur la gouvernance du secteur domestique du bois en proposant des politiques (à mettre en œuvre par la réforme des cadres juridiques), des innovations techniques (par la mise en place des systèmes de garantie de la légalité du bois), un renforcement des capacités des différents acteurs ( $p$. ex. le perfectionnement des compétences et un apport de financement ad hoc aux organisations locales de la société civile pour améliorer les capacités de vérification et de suivi indépendants, et aux médias régionaux et nationaux pour la couverture des questions forestières), et des consultations du public. 


\section{Pro-Formal}

Entre 2010 et 2013, le CIFOR a mis en œuvre Pro-Formal, projet financé par l'UE pour analyser les politiques et réglementations possibles pour que le secteur domestique du bois dans les pays tropicaux soit reconnu et mieux pris en compte. Ce projet s'est déroulé dans trois régions (l'Asie du Sud-Est, l'Afrique subsaharienne et l'Amérique latine) et cinq pays (Cameroun, RDC, Équateur, Gabon et Indonésie). Les résultats montrent que les volumes de bois récoltés par les scieurs artisanaux et la contribution financière du secteur en zone rurale comme en zone urbaine sont considérables, notamment quand on les compare au secteur forestier industriel (Tableau 1).

\section{Tableau 1 : Principales conclusions sur la production formelle et informelle de bois}

\begin{tabular}{|c|c|c|c|c|c|}
\hline & CMR & GAB & RDC & IND & EQU \\
\hline $\begin{array}{l}\text { Consommation } \\
\text { domestique } \\
\text { annuelle } \\
\text { provenant du } \\
\text { sciage artisanal } \\
\text { informel (sciages, } \\
\left.\text { en milliers de } \mathrm{m}^{3}\right)^{\mathrm{a}}\end{array}$ & 662 & 50 & 1024 & $1408^{b}$ & $60-76^{c}$ \\
\hline $\begin{array}{l}\text { Nb d'emplois } \\
\text { informels estimés } \\
\text { (en milliers) }\end{array}$ & 45 & 1 & 25 & $1500^{d}$ & $3,6^{\mathrm{e}}$ \\
\hline $\begin{array}{l}\text { Contribution à } \\
\text { l'économie locale } \\
\text { (en millions } € \text { ) }\end{array}$ & 32 & 1,6 & $34^{f}$ & $63^{9}$ & $9^{h}$ \\
\hline $\begin{array}{l}\text { Bénéfice par } \mathrm{m}^{3} \\
\text { récolté }\left(€ / \mathrm{m}^{3} \mathrm{EBR}\right)\end{array}$ & 5 & 6 & $4-24$ & $85^{i}$ & $7-183$ \\
\hline $\begin{array}{l}\text { Production/ } \\
\text { exportation } \\
\text { annuelles } \\
\text { formelles (sciages, } \\
\text { en milliers de } \mathrm{m}^{3} \text { ) }\end{array}$ & 360 & 150 & 36 & 1 j199 & $170^{k}$ \\
\hline $\begin{array}{l}\text { Taxes forestières } \\
\text { versées par an } \\
\text { (secteur formel, } \\
\text { en millions } €)^{\prime}\end{array}$ & 29,3 & 53 & $58^{\mathrm{m}}$ & $227,2^{n}$ & $6,4^{\circ}$ \\
\hline
\end{tabular}

$\mathrm{CMR}=$ Cameroun, $\mathrm{RDC}=$ République démocratique du Congo, $\mathrm{EQU}=$ Équateur, $\mathrm{GAB}=$ Gabon, IND = Indonésie, $\mathrm{EBR}=$ équivalent bois rond.

a Ceci ne comprend pas le bois vendu sur le marché domestique, mais provenant des scieries industrielles.

b Central Statistical Agency (Institut indonésien de la statistique, données de 2010).

c Cette valeur est basée sur l'estimation de $24 \%$ à $31 \%$ de bois informel coupé dans les forêts naturelles.

d Central Statistical Agency (Institut indonésien de la statistique, 2010). Les données portent sur la filière bois, le papier et les produits dérivés, et le mobilier. Si l'on ne prend en compte que le bois et la filière bois, ce chiffre sélèverait à environ 500000 ouvriers.

e Scieurs artisanaux et ouvriers des usines de transformation compris.

Population rurale uniquement

g Central Statistical Agency (Institut indonésien de la statistique, données de 2010). La contribution à l'économie locale correspond à la rémunération des ouvriers. On part du principe que la moitié de la rémunération des ouvriers en vertu de la classification type des industries (ISIC 16) est versée aux ouvriers des scieries.

h Revenus des petits exploitants agricoles.
Central Statistical Agency (Institut indonésien de la statistique, données de 2010). Mode de calcul : moitié des bénéfices du secteur de I'ISIC 16 divisée par la production de sciages.

j Central Statistical Agency (Institut indonésien de la statistique, 2010).

k FAOSTAT (2012).

I Sauf indication contraire, cela comprend la redevance forestière annuelle, la taxe d'abattage, la taxe d'entrée à la scierie et la taxe d'exportation.

m Redevance forestière annuelle et taxe d'exportation, taxe de déboisement.

ninistère indonésien des Forêts.

- Estimations des auteurs fondées sur la production totale de sciages indiquée par le ministère équatorien de l'Environnement.

Chacun des pays étudiés possède son histoire et sa situation propres qui demandent des politiques spécifiques afin de mieux tenir compte des secteurs régionaux et domestiques du bois dans les cadres juridiques nationaux. Les politiques possibles pour chaque pays sont abordées dans une série d'Infobriefs consultables sur www.cifor.org/pro-formal. Cet Infobrief présente des options politiques découlant d'une analyse comparative de tous les pays étudiés, ainsi qu'un ensemble d'études réalisées dans le cadre du projet sur la formalisation de l'accès aux ressources naturelles et à leur commercialisation (Cerutti et Lescuyer 2011 ; Lescuyer et al. 2011 ; Mejía et Pacheco 2013 ; Lescuyer et al. 2014 ; Putzel et al. 2014 ; Obidzinski et al. sous presse).

\section{Recommandations à propos des politiques}

\section{Veiller au principe d'inclusion politique}

Étant donné les retards dans la mise en œuvre des APV dans la plupart des pays signataires, les attentes sont fortes et donnent lieu à une récente tendance à l'accélération de la délivrance de permis FLEGT. Cependant, cette accélération peut mettre en danger le principe d'inclusion qui a été jusqu'ici une caractéristique positive du processus APV dans tous les pays (Bollen et Ozinga 2013). Les résultats indiquent un fléchissement du principe d'inclusion des instances gouvernementales intersectorielles qui caractérisait les premiers débats sur les APV. L'application de la loi pourrait s'avérer difficile si ces instances sont exclues des phases finales de la mise en œuvre, lors de l'adoption des réformes du cadre juridique relatif aux forêts. Les ministères peuvent se montrer réticents à faire appliquer des règlements à l'élaboration desquels ils n'ont pas contribué, ou même tout bonnement ne pas s'y plier.

L'exclusion de ministères, dont ceux qui supervisent les territoires, l'agriculture, le développement rural et l'emploi, est particulièrement regrettable, car ils pourraient adopter des règlements facilitant la formalisation des petits exploitants et des scieurs artisanaux : transformation des droits d'accès ou d'usage dans les mosaïques paysagères composées de forêt et de petites exploitations où est récoltée la majorité du bois informel.

Pour atténuer ce risque, des ressources devront être affectées à l'organisation de tables rondes plus inclusives. Cependant, les négociateurs des APV auront la mission délicate de trouver le bon équilibre pour ne pas risquer de déclencher un débat avec d'autres ministères qui pourraient, par exemple, faire dévier le discours, de la législation forestière vers d'autres réformes du cadre juridique, comme celles portant sur des lois agraires fondamentales ou le droit foncier. Cela risquerait de retarder le processus de réforme prévu au titre de l'APV de plusieurs années, voire de plusieurs décennies. 

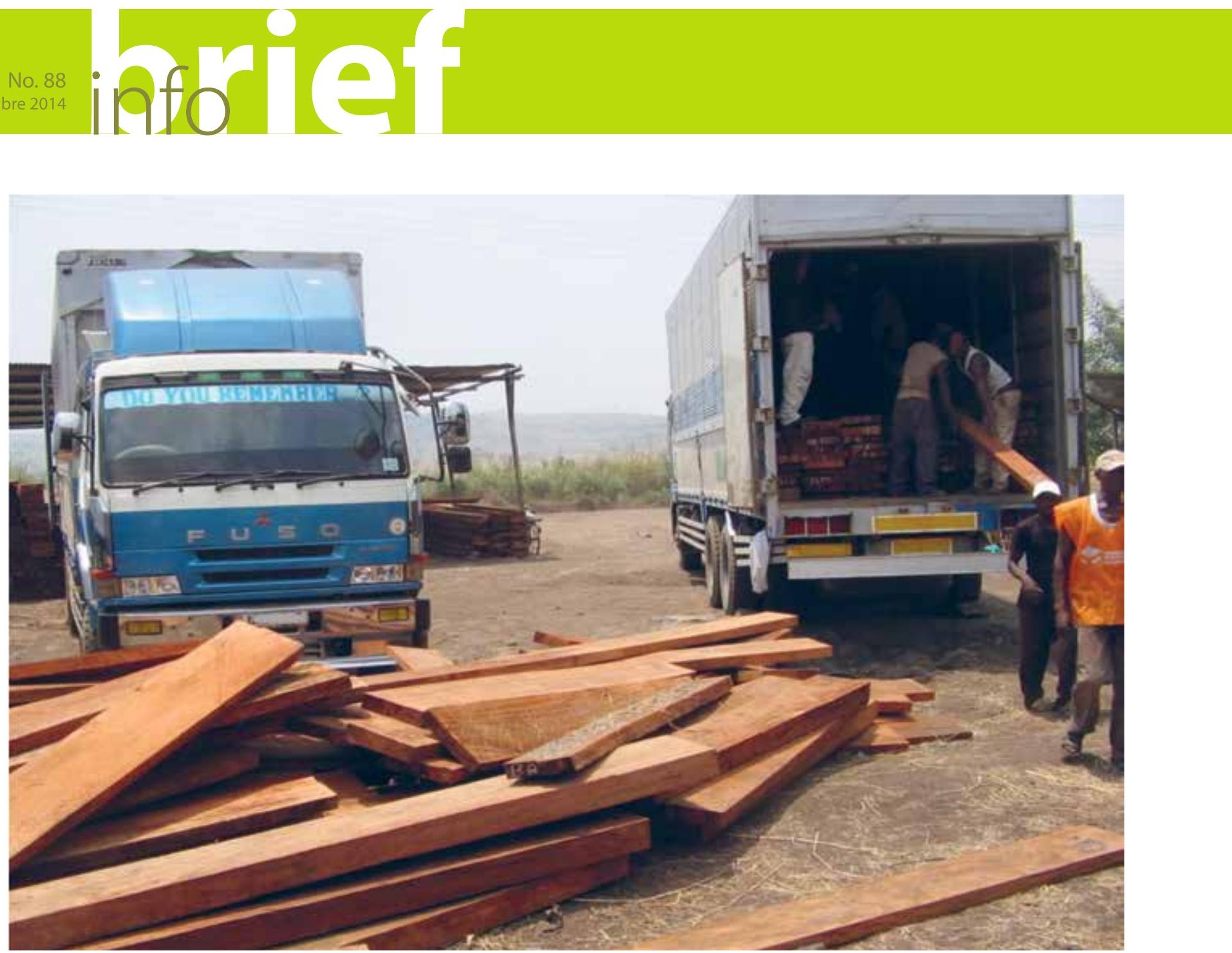

Chargement de bois scié à un poste frontalier, en RDC.

Photo Francois Biloko, Reseau CREF, Goma, DRC

\section{Promouvoir l'inclusion des opérateurs artisanaux}

Toutes les études de cas sur la formalisation entreprises dans le cadre du projet Pro-Formal ont mis en avant le risque d'exclusion, la perte de droits et la possibilité de conflits, surtout à propos des populations marginalisées et disposant de peu de pouvoir. Tout en promouvant des réformes, le processus de mise en œuvre de l'APV devra être particulièrement attentif au risque d'exclusion des exploitants, des scieurs artisanaux et des négociants en bois du pays. Dans les pays concernés par I'APV, les entreprises forestières industrielles, de taille moyenne à grande, jouissent d'un grand avantage sur le plan du lobbying, car elles ont des liens historiques forts avec la classe politique, notamment les ministères en charge des forêts. Comme les APV évoquent le sciage artisanal et les marchés domestiques du bois, il ne faut pas croire cependant que les opérateurs à grande échelle ne feront aucun lobbying au cours de leur mise en œuvre. Ils pourraient le faire afin d'obtenir le plus d'avantages possibles des réformes, notamment au titre de leur situation actuelle « d'entreprises respectant la loi » par rapport aux opérateurs « illégaux » criminalisés.

D’après les résultats livrés par Pro-Formal, les étapes pratiques en vue de mieux promouvoir l'inclusion devront comprendre plusieurs mesures : simplification de la réglementation, droits fonciers plus stables, facilité d'accès au crédit pour une gestion durable des forêts, meilleure information sur les marchés et plus d'action de la part des pouvoirs publics, des bailleurs de fonds et du secteur privé pour aider les exploitants et les scieurs artisanaux à constituer un capital social. En particulier, les études de cas sur la formalisation montrent que les exploitants, les scieurs artisanaux et les négociants nationaux pourraient arriver à se faire entendre grâce à des institutions qui les représenteraient. Des exemples de réussite en matière d'action collective répondant aux coûts élevés de la formalisation existent déjà et donnent des résultats prometteurs. Par exemple, une expérience récente à Jepara en Indonésie montre que des petites et moyennes entreprises ont été capables de mettre leurs ressources en commun pour supporter le coût élevé des permis prévus par le système de garantie de la légalité du bois (Sistem Verifikasi Legalitas Kayu, SVLK) (Purnomo et al. 2014). Cependant, le progrès global de la certification de groupe reste lent.

Planifier des systèmes de vérification attentifs à la demande Dans tous les pays Pro-Formal et dans plusieurs autres, le plus gros problème rencontré par les initiatives actuelles de programmation et de mise en œuvre des systèmes de traçabilité est le grand nombre d'opérations et d'acteurs informels. S'il est bien sûr important de surveiller les activités de récolte de la ressource, afin que le plan d'action FLEGT soit mis en œuvre 
dans les délais et ait plus d'efficacité, il est peu probable que le suivi de vastes étendues forestières soit une solution efficiente et à faible coût. Comme le montrent toutes les études de cas de pays, la grande majorité du bois récolté se retrouve sur les grands marchés ou dans les dépôts des grandes villes. Sans négliger la dimension écologique de la récolte informelle du bois - à laquelle les pouvoirs publics concernés devraient être davantage attentifs (Robiglio et al. 2013) — , au vu de la situation actuelle, des efforts plus importants doivent être faits pour surveiller les activités se trouvant en aval de la chaîne de valeur (p. ex. marchés, dépôts, scieries, secteurs d'activité). Ces derniers devraient aussi assumer en partie la responsabilité technique et financière de la formalisation du fonctionnement de leurs fournisseurs, car ils constituent une cible facile à contrôler pour les administrations limitées par leurs ressources humaines et financières.

Dans le même temps, une aide devrait être fournie (sous forme de réduction d'impôt, d'accès au marché ou d'autres incitations) aux indépendants et aux entreprises, petites ou grandes qui décident d'investir dans les chaînes d'approvisionnement légales, régionales ou nationales.

\section{Simplifier les règles, faire baisser les coûts de transaction et décentraliser les pouvoirs dans la mesure du possible}

Un enseignement important dégagé des pays Pro-Formal est que, pour des raisons à la fois historiques et politiques, les types de permis et les procédures de délivrance afférentes sont bien trop complexes pour que les utilisateurs actuels s'y plient et déposent des demandes. Par conséquent, il est proposé qu'une simplification des types de permis et des procédures de délivrance soit entreprise dans le cadre des réformes juridiques.

La simplification devrait viser une plus grande transparence des procédures de délivrance de permis, du suivi des chaînes de valeur domestiques du bois et de l'accès à l'information sur le prix du bois. La simplification, cependant, devrait aussi nécessairement impliquer une réduction des coûts de transaction pour les exploitants et les scieurs artisanaux. II est inutile d'investir dans des projets de communication en ligne ou mobiles pour la délivrance de permis si le coût de ces permis reste trop élevé pour les operateurs artisanaux.

Une possibilité de réduction des coûts est la décentralisation des principales fonctions et responsabilités d'examen des demandes et de décision d'attribution. II peut s'agir entre autres de la délivrance des permis, de l'accès au crédit grâce à des financements publics, de conseils techniques, ou de séances de formation. Cependant, une réserve non négligeable doit être formulée ici. L'expérience acquise dans plusieurs pays (on dispose d'une bonne documentation sur l'Indonésie et le Ghana) laisse penser que les processus de décentralisation ont souvent été associés à l'augmentation de l'exploitation forestière informelle et des taux annuels nets de déforestation, car les autorités locales

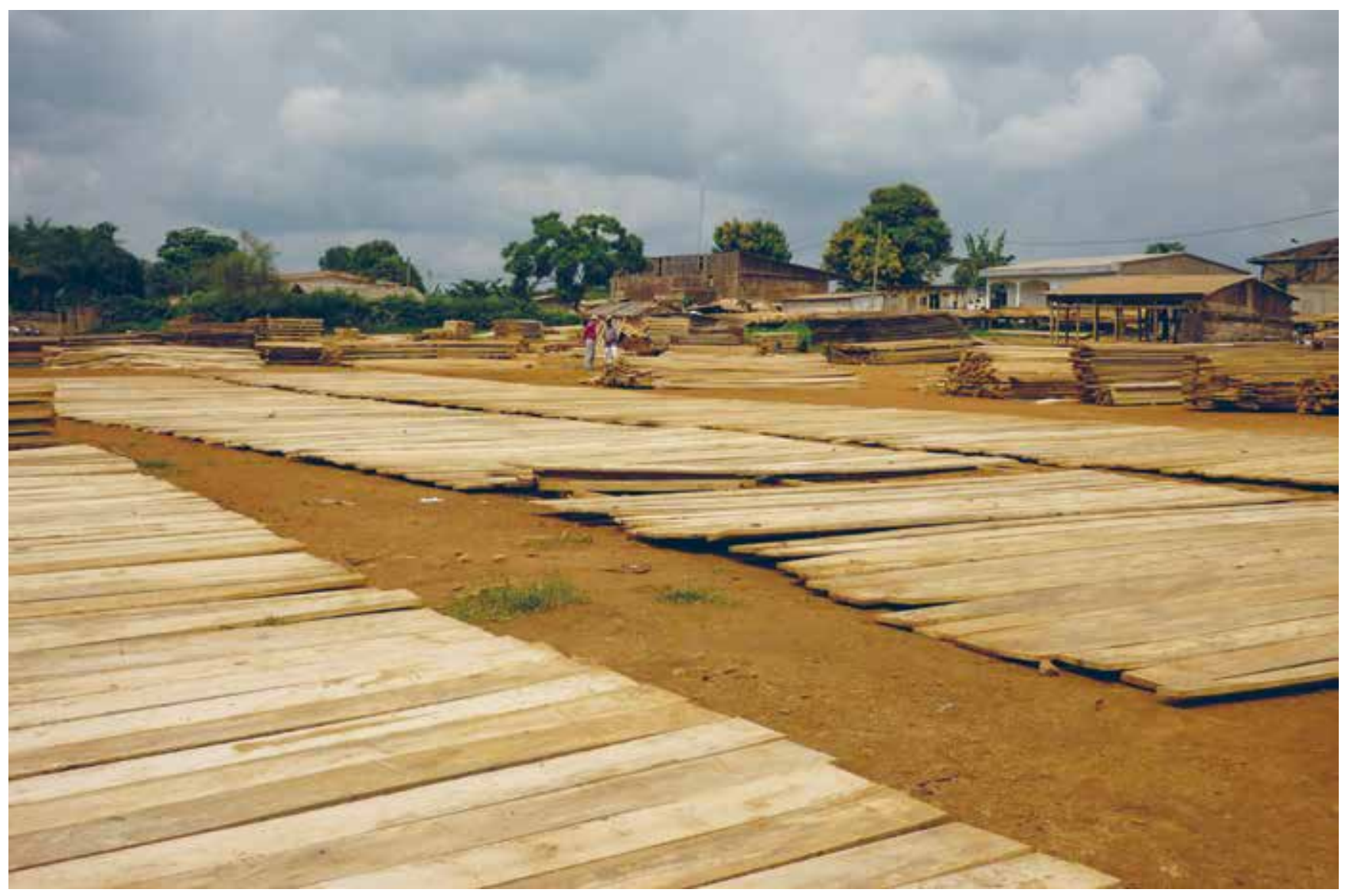

Bois scié prêt à être transporté à une gare, le Cameroun.

Photo Edouard Essiane, CIFOR 
tentent d'accroître leurs recettes (p. ex. Batterbury et Fernando 2006). Les conclusions du projet Pro-Formal ont aussi confirmé le problème persistant de l'accaparement des ressources par les élites, qui doit être pris en compte en cas d'élaboration d'une procédure décentralisée de délivrance de permis.

\section{Adopter des incitations et des régimes de taxation innovants}

Tout processus de formalisation doit laisser aux ministères des Finances une certaine latitude pour mettre en place des régimes de taxation qui tiendront compte de la nature particulière du secteur informel du bois. Ce sera surtout vrai pendant la phase de démarrage des réformes, quand les projets envisagés devront comprendre des incitations fiscales pour motiver les opérateurs informels à intégrer le secteur formel. Par exemple, s'ils sont impliqués judicieusement dans les négociations des APV par les ministères des Forêts, les ministères des Finances pourraient proposer, adopter, mettre en œuvre et assurer le suivi des projets de réduction d'impôt, de "TVA à taux zéro », de " guichet unique pour les investissements », ou des primes pour les fonctionnaires qui saisissent du bois illégal.

Des plans financiers innovants ne devraient cependant être adoptés qu'après l'évaluation des coûts de la mise en œuvre du système de garantie de la légalité du bois pour les opérateurs, quelle que soit leur taille. Une fois qu'auront été estimés les coûts éventuels de la mise en œuvre de ce système et les conséquences potentielles des nouvelles incitations, les négociateurs de l'APV devront débattre d'une feuille de route pour que les opérateurs (grands et petits) se mettent en conformité avec les exigences du futur accord. À ce jour, un très petit nombre de pays APV ont tenu un débat approfondi sur les répercussions financières du fonctionnement du système de garantie de la légalité du bois pour les opérateurs, et surtout les petits. Quand les coûts éventuels du système auront été estimés, des incitations devront être proposées pour aider les opérateurs indépendants et les petites et moyennes entreprises à y faire face (Eba'a Atyi et al. 2013).

Les incitations devraient aussi viser à attirer les opérateurs dans la légalité tout en accroissant leurs capacités de rendementmatière et de transformation en vue d'une meilleure utilisation de la ressource. Des incitations fiscales et financières semblent être le moyen le plus facile d'essayer de réduire le gaspillage. Par exemple, les futures réductions d'impôt officielles pourraient être liées à des investissements dans de meilleures scies, tandis que les connaissances techniques des ministères des Forêts sur l'abondance, la régénération et la gestion de la ressource doivent être mises à profit pour éviter un épuisement rapide, et « légal », de la ressource.

Une autre incitation relativement facile à mettre en œuvre pourrait être l'adoption, dans les pays partenaires, de politiques d'approvisionnement public demandant que tous les marchés publics exigent du bois produit localement dans la légalité. Cela pourrait être l'incitation initiale nécessaire pour que les opérateurs locaux demandent des permis de coupe officiels, tout en faisant face au coût élevé de la procédure, dans l'optique de pouvoir vendre plus cher sur le marché légal.
Pour avoir des effets positifs, la plupart des projets de mesures d'incitations devront aller de pair avec des actions anti-corruption.

\section{Contribuer à l'application de la loi et aux actions anti- corruption}

La simplification et la réforme du cadre juridique ne sont qu'un aspect de la question. L'autre aspect est le grand besoin de faire appliquer la loi avec efficacité. Comme pour d'autres réformes, cela ne devrait pas être exclusivement du ressort des ministères des Forêts. II faudrait en effet également mettre en place un appareil judiciaire efficace et adopter des mesures anticorruption ciblées.

Un système de garantie de la légalité du bois bien conçu et une plus grande transparence pourraient permettre d'éliminer certains foyers de corruption sévissant éventuellement le long de la chaîne de production. Cependant, même le meilleur des systèmes n'empêche pas la fraude. Ceci est d'autant plus vrai quand l'élimination d'une façon de faire ancienne implique la transformation du comportement des fonctionnaires qui subiront des pertes financières, notamment en raison de la formalisation des exploitants et des scieurs artisanaux. Pour que cette transformation ait lieu, la mise en œuvre du système de garantie de la légalité du bois doit s'accompagner d'actions anti-corruption soutenues et clairement affichées dirigées par la présidence ou le cabinet du premier ministre plutôt que par des ministères (ceux des Forêts ou de l'Environnement par exemple).

\section{Surveiller l'application de la nouvelle réglementation}

Si l'application de la loi est fondamentale dans la mise en œuvre du système de garantie de la légalité du bois, le suivi de la réglementation est nécessaire pour que cette application de la loi soit équitable et cohérente et pour éviter une culture de l'impunité susceptible de miner le système. Une mise en application progressive peut être conseillée, les parties les plus «faibles » du secteur, notamment les opérateurs informels actuels, pouvant se voir accorder un délai d'apprentissage avant l'entrée en vigueur et la mise en œuvre d'une nouvelle réglementation.

Cette réglementation doit être juste et sans répercussions disproportionnées pour l'une ou l'autre des parties prenantes. Néanmoins, dans la plupart des pays, les opérateurs informels manquent cruellement de capacités pour appuyer les mesures et se mettre en conformité avec la loi. Si les délais imposés par I'APV sont trop courts, comme l'indiquent les conclusions de Pro-Formal, on pourrait opter pour une démarche qui garantit au départ qu'aucun bois informel n'entrerait dans le système de garantie de la légalité du bois, plutôt que d'essayer d'inclure tous les opérateurs et leurs pratiques actuelles dans le système avant la délivrance des permis FLEGT. Si les permis FLEGT doivent faire leur apparition sous peu sur le marché de l'UE, ce serait l'option la moins dommageable pour les conditions de vie des opérateurs artisanaux, contrairement à une adaptation forcée à des politiques qui ne tiendraient pas suffisamment compte de leur point de vue et de leurs besoins économiques.

\section{Tenir compte des problèmes transfrontaliers}

Le chantier de la formalisation doit être conçu de manière à tenir compte des complexités de la réglementation et de l'application de la loi dans des paysages transfrontaliers. Les 
données historiques associées aux résultats de l'étude indiquent que les marchés du bois régionaux et nationaux sont en croissance constante depuis 10 ans. Les données financières et démographiques disponibles laissent penser que ces tendances vont se poursuivre pendant de nombreuses années. Le bois informel produit actuellement par les exploitants et les scieurs artisanaux compte pour beaucoup sur ces marchés en expansion. Par conséquent, toutes les réformes visant la formalisation du secteur du bois devraient être conçues sans se cantonner au pays, mais en impliquant éventuellement des plateformes ou institutions régionales et internationales (p. ex. le Groupe d'experts sur l'exploitation forestière illégale et le commerce connexe (Experts Group on Illegal Logging and Associated Trade ou EGILAT) dans la région Asie-Pacifique, la Communauté économique des États de l'Afrique centrale, la Communauté est-africaine en Afrique subsaharienne, la Communauté andine, ou l'Organisation du traité de coopération amazonienne en Amérique latine).

À cet effet, les cadres juridiques devraient être adaptés en tant que de besoin pour tenir compte de la possibilité pour les exploitants et les négociants en bois d'exporter leur production. C'est actuellement interdit dans de nombreux pays APV, mais cela pourrait permettre aux opérateurs artisanaux et aux États de saisir des opportunités de marché, avec des retombées directes pour les conditions de vie des opérateurs et les recettes des États. En retour cela encouragerait les transformations nécessaires pour l'entrée sur le marché légal. Des incitations pourraient aussi être adoptées, par exemple en créant au départ un système de quotas d'exportation (normalement assortis de prix supérieurs) pour les petits opérateurs qui se mettraient en conformité.

\section{Perspectives d'avenir}

La signature et la mise en œuvre en cours d'APV dans six pays, ainsi que les négociations et les préparatifs qui ont lieu dans de nombreux autres pays, sont des réussites significatives du processus FLEGT. C'est encore plus le cas dans le contexte de (1) la restructuration permanente (et la nouvelle géographie) du commerce des bois tropicaux au niveau mondial, (2) l'apparition d'autres projets de politiques forestières, dominants et souvent concurrents (notamment la REDD+), et (3) de nouvelles initiatives (p. ex. la loi Lacey et, en Australie, la loi et les règlements sur l'exploitation illégale du bois) et des plateformes de négociation sur des questions semblables (p. ex. EGILAT dans la région AsiePacifique, la Commission des Forêts d'Afrique Centrale (COMIFAC) dans le bassin du Congo et la Communauté de développement d'Afrique australe (SADC) dans le Sud de l'Afrique).

En ce qui concerne les marchés domestiques du bois et les petits opérateurs, de nouvelles politiques publiques et réformes du cadre juridique devront être testées dans des pays sélectionnés, ou dans des régions précises de certains pays, en prenant comme tremplin les engagements clairs pris dans ce sens par des hommes politiques dans les pays VPA ou non, et là où l'on peut faire état d'une expérience passée de réformes de gouvernance du secteur forestier. Actuellement, la phase de mise en œuvre prolongée dans la plupart des pays APV constitue une plateforme optimale pour tester plusieurs des options proposées dans le secteur domestique du bois. Il est possible de dégager des enseignements de certaines initiatives dans des pays non APV, principalement en Amérique latine, où des actions importantes ont été mises en œuvre pour améliorer la gouvernance forestière, y compris des institutions qui soutiennent la gestion forestière et des systèmes plus perfectionnés pour le suivi de la conformité par rapport à la loi.

\section{Bibliographie}

Batterbury SPJ et Fernando JL. 2006 Rescaling governance and the impacts of political and environmental decentralization: An introduction. World Development 34(11):1851-96.

Bollen A et Ozinga S. 2013 Improving Forest Governance: A Comparison of FLEGT VPAs and their Impact. FERN, Bruxelles et Moreton-in-Marsh, Royaume-Uni.

Cerutti PO et Lescuyer G. 2011 The domestic market for smallscale chainsaw milling in Cameroon: Present situation, opportunities and challenges. Document occasionnel 61 Centre de recherche forestière internationale, Bogor, Indonésie. [Version française aussi disponible : Cerutti PO et Lescuyer G. 2011 Le marché domestique du sciage artisanal au Cameroun : État des lieux, opportunités et défis. Document occasionnel 65. Centre de recherche forestière internationale, Bogor, Indonésie.] Eba'a Atyi R, Assembe Mvondo S, Lescuyer G et Cerutti PO. 2013 Impacts of international timber procurement policies on central Africa's forestry sector: The case of Cameroon. Forest Policy and Economics 32:40-48.

Lescuyer G, Cerutti PO, Ndotit Manguiengha S et Bilogo bi Ndong L. 2011 The domestic market for small-scale chainsaw milling in Gabon: Present situation, opportunities and challenges. Document occasionnel 65. Centre de recherche forestière internationale, Bogor, Indonésie. [Version française aussi disponible : Lescuyer G, Cerutti PO, Ndotit Manguiengha S et Bilogo bi Ndong L. 2011 Le marché domestique du sciage artisanal à Libreville, Gabon : État des lieux, opportunités et défis. Document occasionnel 63. Centre de recherche forestière internationale, Bogor, Indonésie.]

Lescuyer G, Cerutti PO, Tshimpanga P, Biloko F, Adebu-Abdala B, Tsanga R, Yembe-Yembe Rl et Essiane-Mendoula E. 2014 The domestic market for small-scale chainsaw milling in the Democratic Republic of Congo: Present situation, opportunities and challenges. Document occasionnel 112. Centre de recherche forestière internationale, Bogor, Indonésie. [Version française aussi disponible : Lescuyer G, Cerutti PO, Tshimpanga P, Biloko F, Adebu-Abdala B, Tsanga R, Yembe-Yembe Rl et Essiane-Mendoula E. 2014 Le marché domestique du sciage artisanal en République démocratique du Congo : État des lieux, opportunités, défis. Document occasionnel 110. Centre de recherche forestière internationale, Bogor, Indonésie.]

Mejía E et Pacheco P. 2014 Forest use and timber markets in the Ecuadorian Amazon. Document occasionnel 111. Centre de recherche forestière internationale, Bogor, Indonésie. [Version espagnole aussi disponible : Mejía E et Pacheco P. 2013. Aprovechamiento forestal y mercados de la madera en la Amazonía Ecuatoriana. Document occasionnel 97. Centre de recherche forestière internationale, Bogor, Indonésie.]

Obidzinski K, Dermawan A, Andrianto A, Komarudin $\mathrm{H}$, Hernawan D et Fripp E. Sous presse. Timber legality verification system and the Voluntary Partnership Agreement in Indonesia: The challenges of the small-scale forestry sector. Document de travail. Centre de recherche forestière internationale, Bogor, Indonésie. 
Purnomo H, Achdiawan R, Melati, Irawati RH, Sulthon, Shantiko B et Wardell A. 2014 Value-chain dynamics: Strengthening the institution of small-scale furniture producers to improve their value addition. Forests, Trees and Livelihoods 23(1-2):87-101.

Putzel L, Kelly A, Cerutti P et Artati Y. 2014 Formalization of natural resource access and trade: Insights from land tenure, mining, fisheries, and non-timber forest products. Centre de recherche forestière internationale, Bogor, Indonésie.

Robiglio V, Lescuyer G et Cerutti PO. 2013 From farmers to loggers: The role of shifting cultivation landscapes in timber production in Cameroon. Small-Scale Forestry 12(1):67-85. doi: 10.1007/s11842-012-9205-3.

\section{Additional reading}

Kishor $\mathrm{N}$ et Lescuyer G. 2012 Controlling illegal logging in domestic and international markets by harnessing multilevel governance opportunities. International Journal of the Commons 6(2):255-70.

Putzel L, Kelly A, Cerutti PO et Artati Y. 2014 Formalization of natural resource access and trade: Insights from land tenure, mining, fisheries, and non-timber forest products. Centre de recherche forestière internationale, Bogor, Indonésie.

Wit M, van Dam J, Cerutti PO, Lescuyer G, Kerrett R et Parker Mckeon J. 2011 Chainsaw milling: Supplier to local markets - A synthesis. Dans : Wit M et van Dam J, éds. Chainsaw milling: Supplier to local markets. Tropenbos International, Wageningen, Pays-Bas. VII-XXII.

Le projet Pro-Formal (EuropeAid/ENV/2010-242904/TPS), financé par I'UE, a été mis en œuvre par le Centre de recherche forestière internationale (CIFOR) dans trois régions (Asie du Sud-Est, Afrique subsaharienne et Amérique latine) et cinq pays (Indonésie, Cameroun, République démocratique du Congo, Gabon et Équateur) de juillet 2010 à décembre 2013. Le projet s'est déroulé avec plusieurs partenaires clés, et certains résultats ont déjà été diffusés par le CIFOR grâce à ses documents occasionnels, Infobriefs, articles publiés dans des revues scientifiques et autres articles sur internet (consultables sur le site internet du projet : www.cifor.org/ pro-formal).

\section{桃 \\ CGIAR \\ RESEARCH PROGRAM ON \\ Forests, Trees and Agroforestry}

Cette recherche a été menée par le CIFOR dans le cadre du Programme de recherche du CGIAR sur les forêts, les arbres et l'agroforesterie (CRP-FTA). Ce programme collaboratif vise à améliorer la gestion et l'utilisation des forêts, de l'agroforesterie et des ressources génétiques des arbres à l'échelle du paysage, des forêts aux exploitations agricoles. Le CIFOR dirige le CRP-FTA en partenariat avec Bioversity International, le CATIE, le CIRAD, le Centre international d'agriculture tropicale et le Centre mondial de l'Agroforestrie.
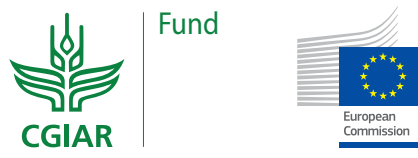